\title{
Allogeneic stem cell transplantation in adult patients with acute myeloid leukaemia and 17p abnormalities in first complete remission: a study from the Acute Leukemia Working Party (ALWP) of the European Society for Blood and Marrow Transplantation (EBMT)
}

Xavier Poiré ${ }^{*}$, Myriam Labopin ${ }^{2,15}$, Johan Maertens ${ }^{3}$, Ibrahim Yakoub-Agha ${ }^{4}$, Didier Blaise ${ }^{5}$, Norbert Ifrah ${ }^{6}$, Gérard Socié ${ }^{7}$, Tobias Gedde-Dhal ${ }^{8}$, Nicolaas Schaap ${ }^{9}$, Jan J. Cornelissen ${ }^{10}$, Stéphane Vigouroux ${ }^{11}$, Jaime Sanz ${ }^{12}$, Lucienne Michaux ${ }^{13}$, Jordi Esteve ${ }^{14 \dagger}$, Mohamad Mohty ${ }^{2,15 \dagger}$ and Arnon Nagler ${ }^{2,16+}$

\begin{abstract}
Background: Acute myeloid leukaemia (AML) with 17p abnormalities (abn(17p)) carries a very poor prognosis due to high refractoriness to conventional chemotherapy, and allogeneic stem cell transplantation (allo-SCT) appears as the only potential curative option.

Methods: To address outcomes after allo-SCT in patients with abn(17p), we retrospectively analysed de novo or secondary AML undergoing SCT between 2000 and 2013 from the EBMT registry.

Results: One hundred thirty-nine patients with confirmed abn(17p) have been selected. At the time of transplant, one hundred twenty-five were in first remission (CR1). Median age was 54 years old. Abn(17p) was associated with a monosomal karyotype in $83 \%$ of patients, complex karyotype in $91 \%$, monosomy 5 or 5 q deletion $(-5 / 5 q-)$ in $55 \%$, monosomy $7(-7)$ in $39 \%$ and both $-5 / 5 q$ and -7 in $27 \%$. Seventy-three patients (59\%) had a reduced-intensity conditioning regimen. The 2-year overall survival (OS) and leukaemia-free survival (LFS) were 28 and 24\%, respectively. The 2-year non-relapse mortality (NRM) was 15\%, and 2-year relapse incidence (RI) was $61 \%$. The cumulative incidence of grade II to IV acute graft-versus-host disease (GvHD) was 24\% and that of chronic GvHD was $21 \%$. In multivariate analysis, the presence of a $-5 / 5 q$ - in addition to abn(17p) was significantly and independently associated with worse OS, LFS and higher RI. Age and donor types did not correlate with outcome. Conditioning intensity was not statistically associated with OS, LFS and NRM when adjusted for patients' age. Conclusions: In contrast to the dismal prognosis reported for AML patients harbouring abn(17p) undergoing conventional chemotherapy, allogeneic SCT provides responses in about 25\% of those patients transplanted in CR1.
\end{abstract} Keywords: Acute myeloid leukaemia, 17p abnormalities, Stem cell transplantation, Survival, First remission

\footnotetext{
* Correspondence: Xavier.Poire@uclouvain.be

${ }^{\dagger}$ Equal contributors

${ }^{1}$ Section of Hematology, Department of Medicine, Cliniques Universitaires

Saint-Luc, 10, avenue Hippocrate, 1200 Brussels, Belgium

Full list of author information is available at the end of the article
} 


\section{Background}

Allogeneic stem cell transplantation (allo-SCT) is now a standard approach recommended for patients with highrisk acute myeloid leukaemia (AML) in remission $[1,2]$. High-risk AML is mainly defined by the presence of determined poor-risk cytogenetic abnormalities at diagnosis together with specific mutational events [3-6]. In general, conventional post-remission high-dose chemotherapy is not capable to eradicate the initiating stem cell leukemic clone of high-risk AML, harbouring strong chemoresistance mechanisms [7], and only the potent graft-versus-leukaemia arising after allo-SCT may overcome the poor prognosis of these high-risk AML subtypes [8]. Indeed, several reports have confirmed the significant advantage of allo-SCT in high-risk AML, especially when performed early in the course of the disease [9-11]. Among the heterogeneous group of high-risk AML, prognosis can be further stratified based on specific genetic abnormalities, and the potential benefit of allo-SCT differs between these diverse AML subtypes [12-16]. While, it is still questionable if distinct genetic abnormalities with a known worse outcome like complex karyotype (CK) and monosomal karyotype (MK) AML will get the same benefit from allo-SCT [17].

TP53 is located in 17p13 chromosomal region and is one of the major tumour suppressor genes, often inactivated by deletion and/or mutation in many tumours [18]. It has been described in 10 to $15 \%$ of AML patients, with an increased frequency in elderly patients and secondary AML [19]. TP53 inactivation is associated in AML with a significantly lower response to intensive chemotherapy, translating into a poor outcome [20]. Although TP53 mutations/deletions show a high correlation with complex karyotype in AML [21-23], TP53 mutations and/or loss have emerged as a strong and independent prognostic marker of very poor outcomes regardless of associated cytogenetic abnormalities [24, 25]. Thus, long-term disease control is observed in less than $5 \%$ of the patients harbouring the TP53 mutations with conventional chemotherapy $[25,26]$. Molecular screening for TP53 mutations is not routinely performed, and loss or disruption of $17 \mathrm{p} 13$ (17p abnormalities, $\operatorname{abn}(17 p))$ is usually identified by FISH analysis [27]. In this context, the potential capability of allo-SCT to overcome the dismal prognosis of abn(17p) AML is of great interest, scarcely explored until now. A first report from Mohr et al. described the outcome of 47 allografted patients and did not show a different outcome compared to non-transplanted patients, raising the hypothesis of a lack of sensitivity of this entity to the potential benefit of graft-versus-leukaemia effect [28]. This detrimental effect of $\mathrm{abn}(17 \mathrm{p})$ on allo-SCT outcomes has been confirmed in another report with an event-free survival (EFS) of only $11 \%$ due to a very high incidence of relapse
[17]. A recent report from Middeke et al. described 201 patients with abn(17p) AML transplanted during the past decade, showing an overall EFS of only $12 \%$, with a slight better outcome among the 84 patients allografted in first complete remission (3-year EFS 18 vs 7\%) $p<$ 0.001) [29]. The purpose of the current study was to explore the potential role of early-phase allo-SCT in $\mathrm{abn}(17 \mathrm{p}) \mathrm{AML}$ in the multicenter, registry context of EBMT, with the aim to identify specific subsets of patients who could benefit from the procedure.

\section{Methods}

\section{Data collection and patient selection}

The data on patients over 18 years of age with a diagnosis of de novo or secondary AML transplanted with a related or unrelated donor were available from the EBMT registry. The latter is a voluntary working group of more than 450 transplant centres reporting regularly on their transplant activity. Only patients having available cytogenetics and transplanted between 1 January 2000 and 31 December 2013 have been selected. Patients with second allo-SCT have been excluded as well as those receiving a haplo-identical transplantation. Audits are routinely performed to insure the quality of the reported data. All patients provided informed consent on the use of their data in retrospective studies. The Review Board of EBMT approved this study. We identified a dataset of 10,799 patients with 5495 patients displaying an abnormal karyotype. All cytogenetic abnormalities have been carefully reviewed by two physicians (Xavier Poiré and Lucienne Michaux). Most centres report conventional karyotype and a few report also FISH results. Cytogenetic results found in the registry are complete or often partial depending on the reporting center. Based on available data, we kept for further analysis only patients for whom data were sufficient to confirm the presence of $\operatorname{abn}(17 p) . A b n(17 p)$ were defined as loss of $17 p 13$ (TP53 locus) such as monosomy 17, deletion (17p), isochromosome $17 \mathrm{q}(\mathrm{i}(17 \mathrm{q}))$, addition $(17 \mathrm{p})$ or other abnormalities that disrupt the $17 \mathrm{p} 13$ locus. Only one center reported a patient with TP53 mutation. Those selected patients have been further categorised as CK, MK, presence of monosomy 7, presence of loss of $5 q$ and/or presence of a inversion of chromosome $3(\operatorname{inv}(3))$. CK has been defined as the presence of 3 or more cytogenetic abnormalities. MK has been defined as two or more autosomal monosomies or one autosomal monosomy in combination with at least one structural chromosomal abnormality. A total of 139 patients from 78 centres met the criteria and have been selected for further analysis.

Myeloablative conditioning (MAC) has been defined as a regimen including total body irradiation (TBI) of more than 8 Gy or a busulfan dose of more than $10 \mathrm{mg} / \mathrm{kg}$. Reducedintensity conditioning (RIC) includes intermediate doses of 
alkylating agents such as $8-10 \mathrm{mg} / \mathrm{kg}$ busulfan, $80-$ $140 \mathrm{mg} / \mathrm{m}^{2}$ melphalan, $600-1200 \mathrm{mg} / \mathrm{m}^{2}$ cyclophosphamide or $5-10 \mathrm{mg} / \mathrm{kg}$ thiotepa, and/or low-dose TBI ( $<3 \mathrm{~Gy})$. The following variables have been selected and included in the analysis: year of transplantation, age, gender, status at transplantation, time to diagnosis to complete remission, time to complete remission to allo-SCT, number of induction courses, type of conditioning regimen, in vivo $\mathrm{T}$ cell depletion, type of $\mathrm{T}$ cell depletion, cytomegalovirus (CMV) status of donor and recipient, donor type, source of stem cells, Karnofsky performance status at transplantation, engraftment, presence of acute and chronic graft-versushost disease (GvHD), grade of acute GvHD, presence of CK, MK, monosomy 7 , loss of $5 \mathrm{q}$ and/or inv(3), cause of death. HLA typing was determined at 10 loci (A, B, C, DRB1, DQB1) by high-resolution techniques, although not all the centres report complete data on HLA. All unrelated donors were defined as HLA matched $(10 / 10)$ or mismatched at 1 locus (9/10). Additional data have been collected on the therapy of relapsing patients when available. HLA data on cord blood (CB) were not captured in this study. Methods and definitions were similar to other studies performed by the Acute Leukemia Working Party of the EBMT [30-32].

\section{Statistical analysis and endpoint definitions}

Endpoints included leukaemia-free survival (LFS), relapse incidence (RI), non-relapse mortality (NRM), overall survival (OS), acute and chronic GVHD and GVHD-free/relapse-free survival (GRFS). All outcomes were measured from the time of stem cell infusion. LFS was defined as survival without relapse; patients alive without relapse were censored at the time of last contact. OS was based on death from any cause. NRM was defined as death without previous relapse. GRFS was defined as survival without grade 3-4 acute GVHD, extensive chronic GVHD, relapse or death. Surviving patients were censored at the time of last contact. The probabilities of OS and LFS were calculated by the Kaplan-Meier test, and those of acute and chronic GVHD, NRM, and relapse by the cumulative incidence estimator to accommodate competing risks. Results are expressed with a 95\% confidence interval (CI). For NRM, relapse was the competing risk, and for relapse, the competing risk was NRM. For acute and chronic GVHD, death without the event and relapse were the competing risks.

For all prognostic analyses, continuous variables were categorised and the median was used as a cut-off point. A Cox proportional hazards model was used for multivariate regression. Factors associated with a $p$ value less than 0.05 by univariate analysis were included in the model. Results were expressed as hazard ratio (HR) with 95\% confidence interval
All tests were two-sided. The type 1 error rate was fixed at 0.05 for determination of factors associated with time to event outcomes. Statistical analyses were performed with SPSS 19 (SPSS Inc./IBM, Armonk, NY) and R 3.0.1 (R Development Core Team, Vienna, Austria) software packages.

\section{Results}

\section{Patients' characteristics}

A total of 139 patients with abn(17p) have been identified. There were 125 patients transplanted in first complete remission (CR1), while only 14 patients were transplanted in second remission (CR2). Because of the small number of patients transplanted in CR2, further analysis has been focused on CR1 patients. A detailed table of the different $a b n(17 p)$ is available as a Additional file 1: Table S1.

A total of 125 patients with abn(17p) transplanted in CR1 have been analysed in November 2015. The median follow-up of the cohort was 21 months (ranging 3146 months). The median age at transplantation was 54 years old (ranging 18-69 years old). The median year of transplantation was 2009 (ranging 2000-2013). The median time from diagnosis to CR1 was 57 days (ranging 18-170 days), and median time from CR1 to transplantation was 82 days (ranging 11-286 days). For 81 patients, we had information about the number of induction courses to reach CR1. Fifty-two had just one course, 27 had 2 and 3 needed 3 rounds of chemotherapy. Most patients were de novo AML (85\%) and only 19 patients corresponded to secondary AML, seven of them arising from an antecedent myelodysplastic syndrome. The majority of patients were male $(57 \%)$ and were transplanted with a Karnofsky performance status of more than $90 \%$ (70\%). A sibling donor was used in $48 \%$ and an unrelated donor in 43\% (10/10: $N=23$ (64\%); 9/10: $N=13$ (33\%), missing: $N=36$ ) whereas a cord blood was used in 10 patients. Source of stem cell was mostly peripheral blood (76\%). CMV status was positive in $68 \%$ of the patients and also $68 \%$ of the donors. Fifty-one patients received a MAC and 74 patients a RIC. In patients less than 50 years old, only 14 patients (26.4\%) received a RIC but this number increased up to $60(83.3 \%)$ in patients over 50 years old $\left(p<10^{-5}\right)$. Most frequent MAC were the combination of cyclophosphamide and busulfan $(N=19)$ followed by the association of total body irradiation (TBI) with cyclophosphamide $(N=14)$. Most RIC were fludarabine and TBI $(N=25)$ closely followed by fludarabine and busulfan $(N=24)$. Seven patients received the sequential FLAMSA-RIC approach [33]. In vivo $\mathrm{T}$ cell depletion has been used in 64 patients. Among those, 47 patients received antithymocyte globulin (ATG) and 17 patients alemtuzumab. Regarding associated cytogenetic categories, most 
patients carried also a CK $(N=98)$ or a MK $(N=86)$. An inv(3) was present in only 3 patients. Monosomy $7(-7)$ was seen in 41 patients and a monosomy 5 or a loss of $5 \mathrm{q}(-5 / 5 \mathrm{q}-)$ in 58 patients. Both -7 and $-5 / 5 \mathrm{q}$ - were present together in 28 patients. Table 1 summarises the patients' characteristics.

Table 1 Patients' characteristics $(N=125)$

\begin{tabular}{ll}
\hline Median age at SCT (range) & 53.6 years old (18-69) \\
Median follow-up (range) & 21 months (3.3-146) \\
Interval between diagnosis and CR1 (range) & 56.5 days (18-170) \\
Intervals from CR1 to SCT (range) & 81.5 days (11-286) \\
Median year of SCT & $2009(2000-2013)$ \\
Secondary AML, N (\%) & $19(15.2 \%)$ \\
CMV+ patient, N (\%) & $84(68.3 \%)$ \\
CMV+ donor, N (\%) & $65(68.3 \%)$ \\
Karnofsky >90\% at SCT, N (\%) & $83(70.3 \%)$ \\
Gender, N (\%) & \\
Male & $71(57 \%)$ \\
Female & $54(43 \%)$ \\
Donor type, N (\%) & \\
Sibling & 20 \\
Unrelated & $60(48 \%)$ \\
Cord blood & $54(43.2 \%)$ \\
Source of SC, N (\%) & $10(8 \%)$ \\
BM & 20 \\
PB &
\end{tabular}

Abbreviations; $N$ number, $C R 1$ first complete remission, $S C T$ stem cell transplantation, $A M L$ acute myeloid leukaemia, $C M V$ cytomegalovirus, $B M$ bone marrow, $P B$ peripheral blood, $C B$ cord blood, $M A C$ myeloablative conditioning, $R I C$ reduced-intensity conditioning, $A T G$ anti-thymocyte globulin

\section{Engraftment and graft-versus-host disease}

Engraftment was successful in 117 patients (94\%). Six patients showed a graft failure and 1 patient lost his graft. The cumulative incidence of grade II to IV acute GvHD was $24 \%$. The 2-year cumulative incidence of chronic GvHD was only 21\% [95\% CI 14.2-29.5] (Fig. 1). This low incidence is explained because many patients relapsed before developing chronic GvHD. A Cox proportional hazards model including age, donor type, use of ATG during the conditioning regimen, source of stem cells and conditioning intensity has been performed for cGvHD (Table 2). Only the use of ATG during the conditioning regimen was significantly associated with less chronic GvHD (HR 0.33, 95\% CI $0.11-0.97 ; p=0.04$ ), whereas donor other than an HLA-matched sibling showed just a trend towards more chronic GvHD $(p=0.07)$.

\section{Non-relapse mortality and relapse incidence}

The 2-year cumulative incidence of NRM was 15\% [95\% CI 8.9-21.8] as illustrated in Fig. 2a. None of the analysed variables (i.e. conditioning intensity, in vivo $\mathrm{T}$ cell depletion, use of ATG, age, donor or patient gender, female donor to male recipient, Karnofsky performance status, donor type, number of induction course, CK, MK, $-7,-5 / 5 q$ - or both -7 and $-5 / 5 q-)$ were significantly associated with NRM neither in univariate analysis nor in multivariate analysis.

Seventy-six patients relapsed at a median interval of 4 months from allo-SCT (range 0.2-92.8 months) translating into a 2-year cumulative incidence of relapse of 61.3\% [95\% CI 51.5-69.7] as illustrated in Fig. 2b. The 2 factors significantly associated with a higher RI were conditioning intensity and presence of $-5 / 5 q-$. On the

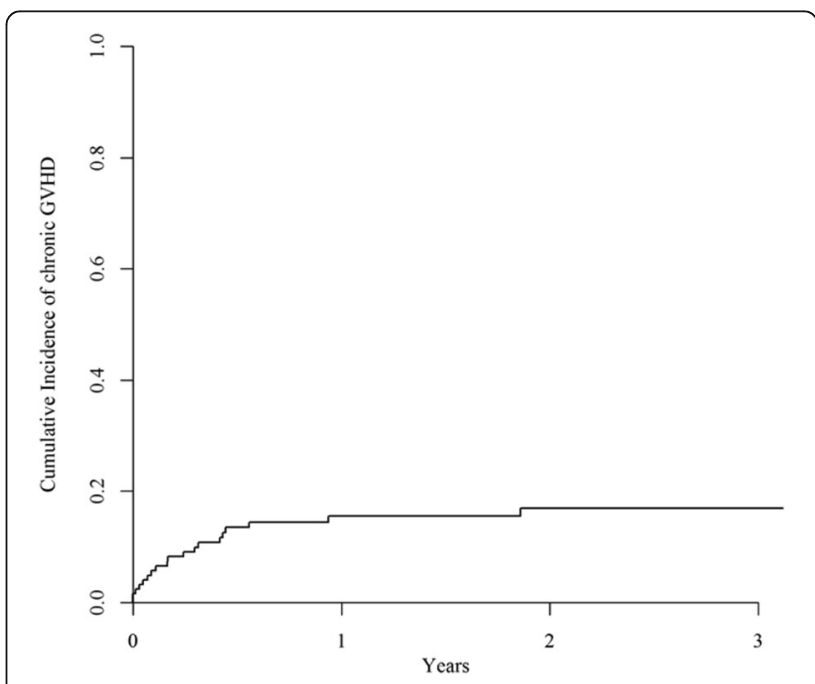

Fig. 1 Cumulative incidence of chronic GvHD. The 2-year cumulative incidence of chronic GvHD was 21\% [95\% Cl 14.2-29.5] 
Table 2 Multivariate analysis using a Cox proportional hazards model, $N=96$. Chronic GvHD

\begin{tabular}{lllll}
\hline & $p$ & HR & $95 \% \mathrm{Cl}$ & \\
\hline Age $\geq 50$ years old & 0.41 & 1.51 & 0.56 & 4.06 \\
Donor other than MSD & 0.07 & 2.39 & 0.92 & 6.2 \\
ATG vs No & 0.04 & 0.33 & 0.11 & 0.97 \\
PB vs BM & 0.89 & 0.92 & 0.31 & 2.71 \\
RIC vs MAC & 0.12 & 2.34 & 0.8 & 6.86 \\
\hline
\end{tabular}

Abbreviations: MSD matched sibling donor, ATG anti-thymocyte globulins, $P B$ peripheral blood, $B M$ bone marrow, $R I C$ reduced-intensity conditioning, $M A C$ myeloablative conditioning, $\mathrm{HR}$ hazard ratio, $\mathrm{Cl}$ confidence interval

contrary, in vivo $\mathrm{T}$ cell depletion was not associated with higher relapse rate. The relapse incidence was 53\% [95\% CI 37-66.2] after a MAC and $68 \%$ [95\% CI 55.2-78.2] when a RIC was used $(p=0.01)$. The presence of $-5 / 5 q-$ was associated with a significant higher RI $(70 \%[95 \%$ CI
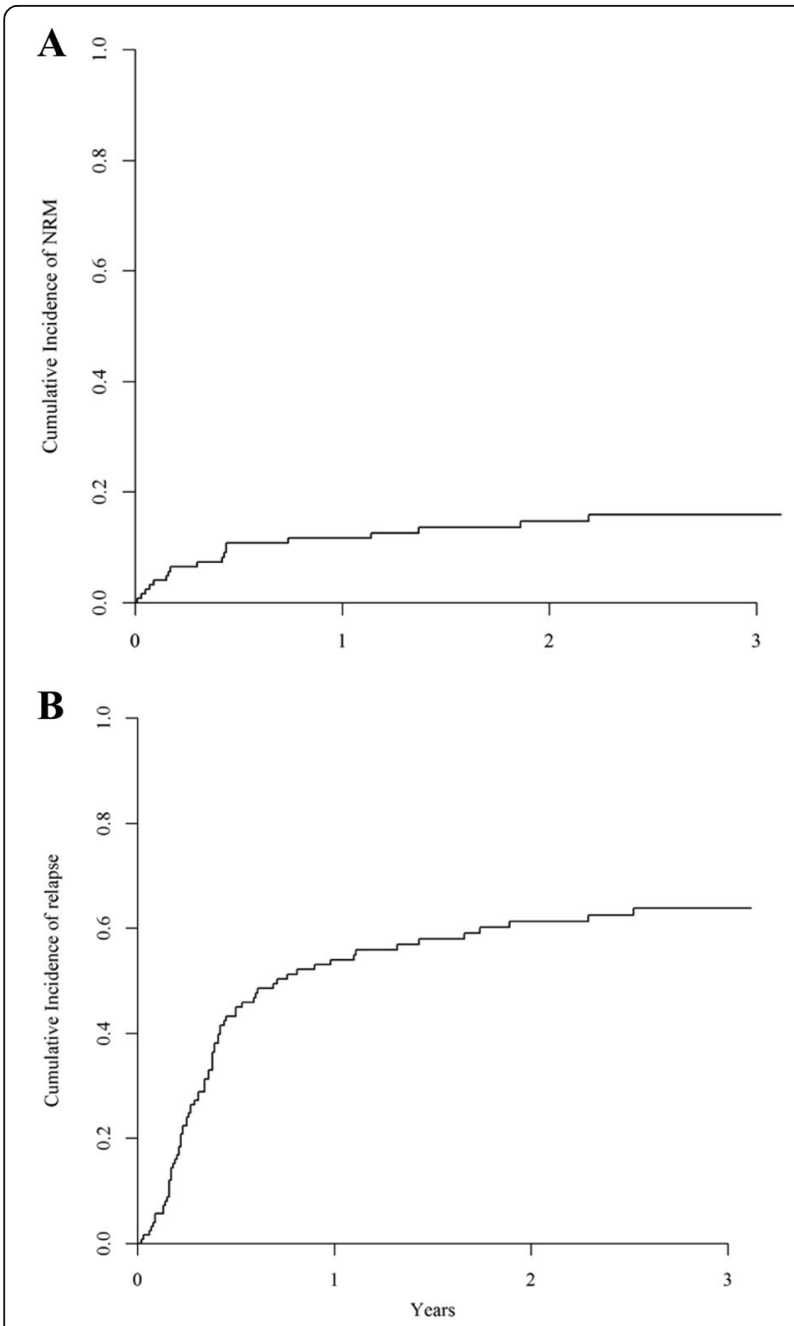

Fig. 2 Non-relapse mortality (NRM) (a) and relapse incidence (RI) (b). The 2-year cumulative incidence of NRM was 15\% [95\% Cl 8.9-21.8] (a) and the 2-year cumulative incidence of relapse was 61.3\% [95\% Cl 51.5-69.7] (b)
55.4-80.9]) compared to patients without $-5 / 5 q-(51 \%$ [95\% CI 35.4-65.4], $p=0.03)$. Patients' age above 50 years old and MK showed only a trend towards a higher RI ( $p$ $=0.06$ and $p=0.05$, respectively). The number of induction courses to reach CR1 did not impact on relapse rate. In multivariate analysis, only the presence of $-5 / 5 q-$ kept its significant impact on RI $(p=0.03)$ while conditioning intensity, age and MK did not show a significant impact on relapse risk (Table 3). Among the 56 relapsed patients with available information, 16 patients received donor leukocyte infusion (DLI). Thirteen of them received a second allo-SCT thereafter and 2 additional patients received a second allo-SCT as the only cell-based therapy for relapse. The 2-year probabilities of OS were 8.4\% after DLI [95\% CI 0-24.1] and 20\% after second allo-SCT [95\% CI 0-55.1].

\section{Survival}

The 2-year probability of OS was $28 \%$ [95\% CI $19.7-$ 37.1] (Fig. 3a). In univariate analysis, factors significantly associated with a worse OS were RIC, older age, MK and presence of $-5 / 5 q$ - Monosomy 7 showed only a trend towards a decreased OS $(p=0.06$ and $p=0.08$, respectively). Thus, the 2-year probability of OS was $40 \%$ [95\% CI 25-55] after a MAC and 21\% after a RIC [95\% CI 10-31] $(p<0.005)$. Patients above 50 years old had a worse OS (22\%, [95\% CI 12-36]) than younger patients (39\%, [95\% CI 23-54], $p<0.005)$. Given the strong interaction between use of RIC and older age, as previously described, conditioning intensity did not show any impact on OS when adjusted for age. Concerning associated cytogenetic categories, patients harbouring a MK had a decreased 2-year OS (19\%, [95\% CI 17-36]) compared to patients without this cytogenetic abnormality

Table 3 Multivariate analysis using a Cox proportional hazards model, $N=90$. Only variables with $p<0.05$ in univariate analysis. LFS, OS and RI

\begin{tabular}{llllll}
\hline & $p$ & HR & $95 \% \mathrm{Cl}$ & \\
\hline LFS & Age $\geq 50$ years old & 0.48 & 1.23 & 0.69 & 2.20 \\
& RIC vs MAC & 0.13 & 1.54 & 0.89 & 2.68 \\
& MK & 0.21 & 1.57 & 0.77 & 3.19 \\
& Monosomy 5q & 0.02 & 1.83 & 1.09 & 3.07 \\
OS $\quad$ Age $\geq 50$ years old & 0.35 & 1.37 & 0.71 & 2.64 \\
& RIC vs MAC & 0.07 & 1.75 & 0.95 & 3.24 \\
& MK & 0.14 & 1.79 & 0.82 & 3.91 \\
& Monosomy 5q & 0.01 & 2.02 & 1.18 & 3.47 \\
RI & Age $\geq 50$ years old & 0.54 & 1.21 & 0.65 & 2.25 \\
& RIC vs MAC & 0.13 & 1.58 & 0.87 & 2.88 \\
& Monosomy 5q & 0.03 & 1.84 & 1.06 & 3.19 \\
\hline
\end{tabular}

Abbreviations: $N$ number, LFS leukaemia-free survival, $O S$ overall survival, $R I$ relapse incidence, $H R$ hazard ratio, $C l$ confidence interval, $M A C$ myeloablative conditioning, RIC reduced-intensity conditioning, $M K$ monosomal karyotype 

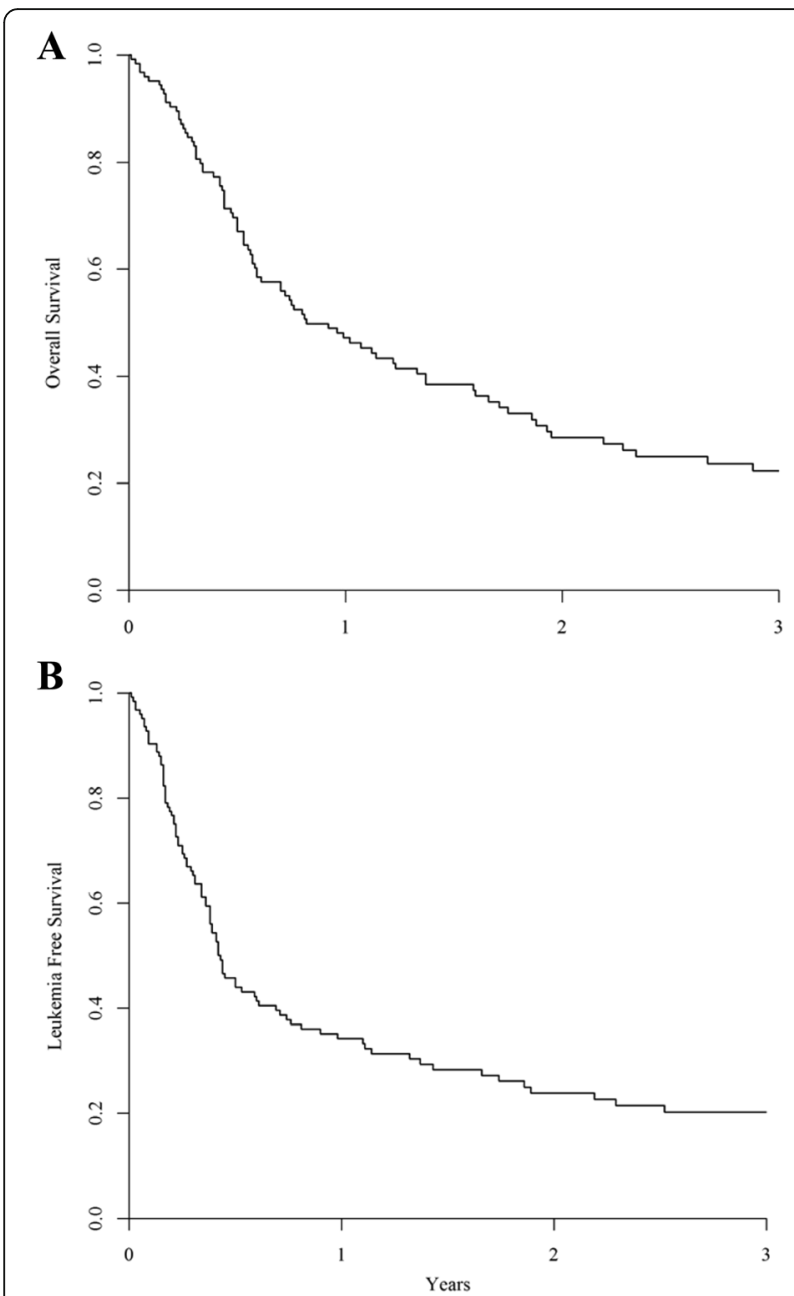

Fig. 3 Overall survival (OS) (a) and leukaemia-free survival (LFS) (b) In the whole cohort, the 2-year probability of OS was $28 \%[95 \% \mathrm{Cl}$ 19.7-37.1] (a) and the 2-year probability of LFS was 24\% [95\% Cl 15.7-31.9] (b)

(58\%, [95\% CI 34-82], $p=0.005)$ as well as patient with concomitant $-5 / 5$ q- $(12 \%$, [95\% CI 2-22] vs $44 \%$, [95\% CI 28-59], $p<0.005)$. Taking together -7 and $-5 / 5 q-$ status, absence of $-5 / 5 q$ - was associated to a better outcome (2-year OS 47\% [95\% CI 30-65] and 31\% [95\% CI 0-63] in patients without and with concomitant -7 , respectively) compared to the subset of patients harbouring -5/5q- (2-year OS 16\% [95\% CI 1-31] and 10\% [95\% CI 0-22], according to simultaneous -7 or not, respectively) (Fig. 4a). Thus, in multivariate analysis, the presence of $-5 / 5 q$ - was significantly associated with a decreased OS (HR 2.02; 95\% CI 1.2-3.5, $p=0.01$ ), whereas RIC was associated with a trend towards a worse OS $(p=0.07)$ (Table 3$)$. Causes of death were disease related in 63 patients, infections in 13, GvHD in 11, haemorrhage in 1 and others in 2 patients.

The 2-year probability of LFS was $24 \%$ [95\% CI 15.7-31.9] as illustrated in Fig. 3b. In univariate
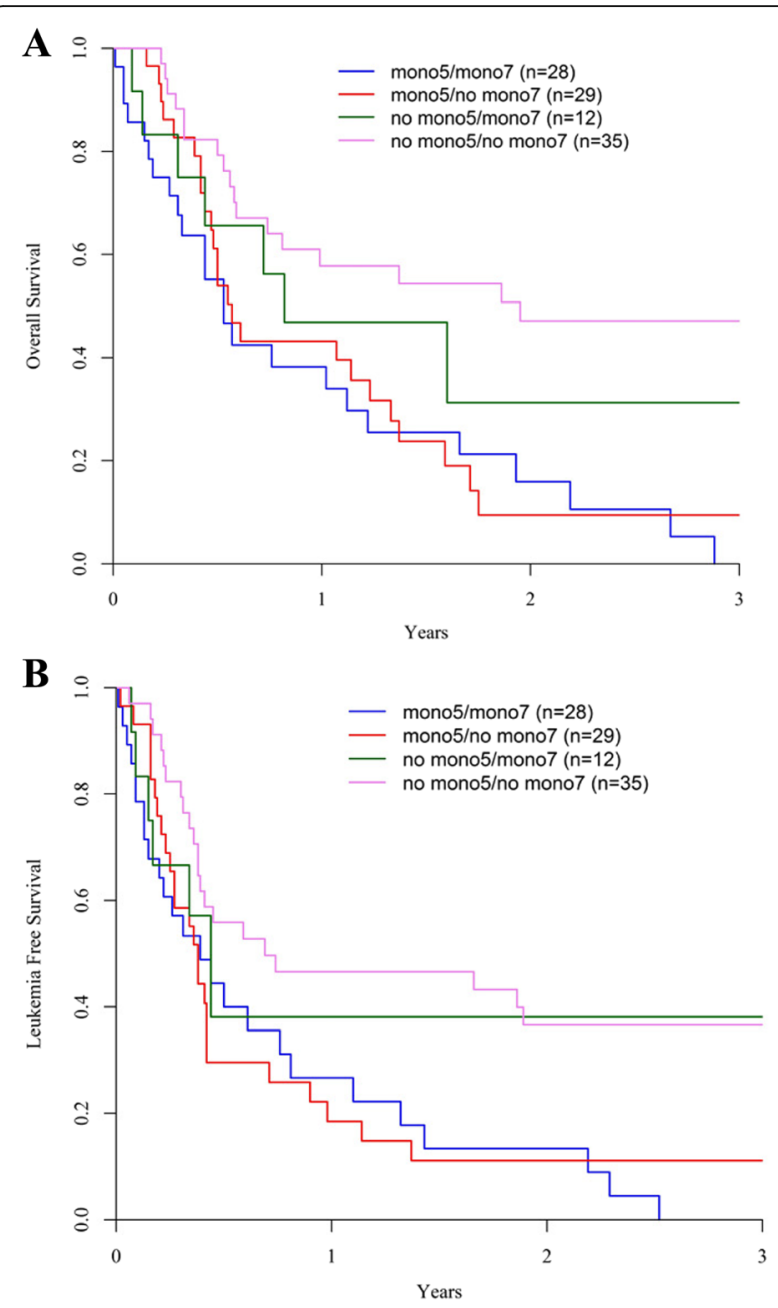

Fig. 4 Overall survival (OS) (a) and leukaemia-free survival (LFS) (b) by cytogenetics subgroup. Mono5 refers as the presence of monosomy 5 or loss of $5 q$ and mono 7 refers as the presence of monosomy 7. Absence of Mono5 was associated to a better OS (2-year OS $47 \%$ [95\% Cl 30-65] and 31\% [95\% Cl 0-63] in patients without and with mono7, respectively) compared to the subset of patients harbouring mono5 (2-year OS: $16 \%$ [95\% Cl 1-31] and 10\% [95\% Cl 022], according to simultaneous mono7 or not, respectively) (a). The deleterious impact of mono5 on LFS was independent of presence of additional mono7, with a 2-year LFS of $11 \%$ [95\% Cl 0-23] (mono5 without mono7) and 13\% [95\% Cl 0-27] (mono5 with mono7) vs 38\% [95\% Cl 9-67] (absence of mono5 with mono7) and $37 \%$ [95\% Cl 20-53] (absence of both abnormalities, $p=0.007$ ) (b)

analysis, decreased LFS was significantly associated with RIC (2-year LFS of $20 \%$ [95\% CI 8-29] and $30 \%$ [95\% CI 16-44] after RIC and MAC allo-SCT, respectively, $p=0.01)$, age above 50 years old (2-year LFS: $20 \%$ [95\% CI 10-30] vs $29 \%$ [95\% CI 15-43], $p<$ 0.005), MK (2-year LFS $17 \%$ [95\% CI 8-25] vs $49 \%$ [95\% CI 25-72], $p=0.02$ ), and presence of $-5 / 5 q-)$. The deleterious impact of $5 \mathrm{q}$ loss was independent of presence of additional -7 , with a 2 -year LFS of $11 \%$ [95\% CI 0-23] (-5/5q- without -7) and 13\% [95\% CI 
0-27] (-5/5q- with -7) vs 38\% [95\% CI 9-67] (absence of -5/5q- with -7) and 37\% [95\% CI 20-53] (absence of both abnormalities, $p=0.007$; Fig. $4 \mathrm{~b}$ ). In vivo $\mathrm{T}$ cell depletion and the use of ATG were not significantly associated with worse LFS. In multivariate analysis, only the presence of $-5 / 5 q$ - remained significantly associated with a decreased LFS $(p=0.02)$ (Table 3$)$.

The presence of chronic GvHD was associated with significantly decreased risk of RI, but resulted in higher NRM, and worse OS and LFS $(p<0.005)$ (Table 4$)$. The 2-year probability of GvHD and relapse-free survival (GRFS) was 16\% [95\% CI 9-23]. Among the 10 patients transplanted with $\mathrm{CB}$, we found similar 2-year OS and LFS of $27 \%$ [95\% CI 0-56] and 27\% [95\% CI 2-58], respectively. No significant differences were found with the other patients ( $p=0.95$ and $p=0.81$, respectively). In this small cohort, four out of them showed the combination of $\operatorname{abn}(17 p)$ and $-5 / 5 q-$.

\section{Discussion}

P53 loss of function resulting from chromosomal losses of 17p region and TP53 gene mutations result in marked chemorefractoriness and very poor prognosis, with virtually incurability for most patients treated with conventional AML chemotherapy [24, 25, 28, 29]. The present study focused on the capability of allo-SCT to circumvent this dismal prognosis. In patients allografted in CR1, LFS at 2 years was $24 \%$, suggesting the potential curability of a proportion of these patients with this approach, and the existence of a potent graft-versustumour effect capable to sustain response. Our cohort might correspond to a highly selected patient population, with some degree of chemosensitivity sufficient to achieve an initial response, and is therefore not representative of the whole abn(17p) AML. However, these results confirm the role of allo-SCT as a reasonable option for the subset of patients achieving sufficient cytoreduction at the time of transplantation. $A b n(17 p)$ are highly represented in overlapping cytogenetically very high-risk AML, such as MK and CK, and might participate in the underlying mechanisms responsible of the their refractoriness to standard intensive AML therapy.

Table 4 Multivariate analysis using a Cox proportional hazards model. Impact of cGvHD on outcomes (time-dependant variable)

\begin{tabular}{lllll}
\hline & $p$ & HR & $95 \% \mathrm{Cl}$ & \\
\hline RI & $<10^{-4}$ & 0.76 & 0.69 & 0.85 \\
NRM & $<10^{-4}$ & 2.92 & 2.62 & 3.25 \\
LFS & $<10^{-4}$ & 1.45 & 1.35 & 1.56 \\
OS & $<10^{-4}$ & 1.25 & 1.17 & 1.35 \\
\hline
\end{tabular}

Abbreviations: $N$ number, LFS leukaemia-free survival, OS overall survival, $R /$ relapse incidence, NRM non-relapse mortality, $H R$ hazard ratio, $\mathrm{Cl}$ confidence interval
Nonetheless, the study provides evidences of the urgent unmet need to develop novel strategies for these patients. Different transplant modalities, concerning donor source or conditioning regimen, did not have a major impact on transplant outcome in our study, and future improvement attempts must explore pre- and posttransplant interventions, together with innovative modifications of allo-SCT conditioning regimen.

Our results are quite comparable to those reported by Middeke et al. [29]. The 2-year LFS and OS of 24 and $28 \%$, respectively, in our cohort are more favourable compared to the previous retrospective study from Mohr et al., based on 47 transplanted patients, which did not show any advantage compared to conventional therapy, with a 4-year probability of survival for the entire cohort of only 4\% [28]. Relapse was the main cause of treatment failure, achieving $70 \%$ at 2 years after RIC conditioning, and these relapses occurred at a median interval from transplant of 4 months, indicating the need of implementing early interventions in the post-transplant period to prevent relapse. Notably, the current results in patients with AML harbouring abn(17p) are similar to those observed with MK and CK AML. In those studies, an independent effect of $\mathrm{abn}(17 \mathrm{p})$ has not been found [13-15]. In fact, genomic losses of $17 \mathrm{p}$, together with losses of $5 q$ and $7 q$, and gains $11 q$ and $8 q$, are the most frequent cytogenetic abnormalities described in CK [34]. Nevertheless, our study cohort represents a more homogeneous population than the one addressed in the studies evaluating MK and CK AML. On the other hand, we were not able to find a significant effect of the presence of MK, probably because $83 \%$ of patients displayed MK at diagnosis.

NRM was only $17 \%$, probably reflecting the positive selection effect in this population, enriched with responsive patients to previous chemotherapy. In fact, these 139 patients represent only $1.3 \%$ of 10,799 patients with an available karyotype in the EBMT database, a lower proportion than the expected rate of $5-10 \%$ in general AML population [19]. These $1.3 \%$ of patients refer only to the proportion of $\mathrm{abn}(17 \mathrm{p})$ AML patients who were in remission and fit enough to survive until the transplantation procedure. Lower intensity conditioning regimens were associated to a higher relapse risk, up to $70 \%$, in the univariate analysis, an association not confirmed in the multivariate analysis adjusted for other variable such as concomitant presence of $5 \mathrm{q}$ loss and age. Nonetheless, the effect of different regimens aimed to enhance antitumour effect without increasing toxicity must also be explored in the next future.

Presence of chronic GvHD, analysed as timedependant variable, was independently associated with a lower relapse risk (HR 0.76), supporting the existence of a genuine and potent graft-versus-leukaemia. This 
antitumour effect of chronic GvHD, nonetheless, did not result in a neat benefit due to its association to a higher NRM translating into worse OS and LFS. Recognition of a potential graft-versus-leukaemia effect in abn(17p) AML would give the basis to develop strategies aimed to harness this alloimmune effect in the early posttransplant period, such as early withdrawal of immunosuppression or administration of prophylactic donor leukocyte infusion $[35,36]$. Post-transplant administration azacytidine might contribute to stimulate the antitumour donor graft effect by enhancing the expression of tumour and minor histocompatibility antigens, with the theoretical added advantage of avoiding an increased GvHD rate by expansion of $\mathrm{T}$ regulatory cell population. Several studies have demonstrated feasibility of azacytidine during the post-transplant period, and the correlation of the expansion determined cytotoxic $T$ cell subsets against tumour antigens with a lower relapse incidence, but the clinical benefit of such strategy should be further proven [37-40]. Other innovative donor cell strategies such as NK cell infusion or Cytokine-induced killer population, with a theoretical lower potential of GvHD induction, must be of high interest in this setting [41-43]. Anyhow, based on the very short median time to relapse, post-transplantation interventions should be given early as a prophylactic or maintenance strategy. Vosaroxin $[44,45]$ is a quinolone derivative reported to be TP53 independent and shows some clinical benefit in combination with high-dose cytarabine in relapsed patients. Currently, it is completely unknown if this agent, administered prior to allo-SCT, might result into improved outcome after allo-SCT, but it might constitute a model to bring abn(17p) AML with better response to allo-SCT.

Additional chromosomal $5 \mathrm{q}$ loss conferred an even worse outcome in this cohort of patients, with an increased relapse risk and 2-year OS and LFS of only 10 and $11 \%$, respectively, regardless the presence of concomitant monosomy 7. It was the only independent prognostic factor in this patient population. While the biological basis accounting for this combined deleterious effect is mostly unknown, TP53 mutations have been frequently observed in association with loss of $5 \mathrm{q}$, up to $80 \%$ of cases in some series, suggesting cooperation between TP53 mutations and loss of putative tumour suppressor genes localised in 5q region [46-48]. Previous reports supported this hypothesis that multiple candidate genes localised on 5q cooperate with TP53 mutations in the pathogenesis of myelodysplastic syndrome or AML [49-51]. Many genes on 5q have been proposed, but recently, haploinsufficiency of $E R G 1$ and $A P C$ in combination with the early acquisition of TP53 mutations have emerged as a potential mechanism leading to the development of a leukemic clone resistant to apoptosis and with increased genomic instability [48]. This translates into chemoresistance and worse outcomes confirmed in our study with patients harbouring both abn(17p) and -5/5q-. In this subgroup of patients, the benefit of allo-SCT appears very limited and new therapeutic strategies are strongly warranted. On the contrary, patients with abn(17p) without $-5 / 5 q$ - showed a relative good outcome after allo-SCT with a 2-year probability of LFS of $37-38 \%$.

\section{Conclusions}

Allo-SCT arises as the best therapeutic option to improve survival in selected patients harbouring abn $(17 \mathrm{p})$ and achieving $\mathrm{CR}$ after frontline chemotherapy, especially in the absence of $-5 / 5 q-$. Nonetheless, clinical benefit of allo-HCT remains very limited, followed by a high relapse incidence. Recognition of a potential graftversus-leukaemia effect preventing relapse in some patients gives the rationale basis for the development of early chemotherapy-based or cell-based strategies to prevent relapse and therefore to increase the potential benefit of allo-SCT in these patients.

\section{Additional file}

Additional file 1: Table S1. (DOCX $13 \mathrm{~kb})$

\begin{abstract}
Abbreviations
-5/5q-: Monosomy 5 or loss of 5q-; -7: Monosomy 7; Abn(17p): 17p abnormalities; Allo-SCT: Allogeneic stem cell transplantation; AML: Acute myeloid leukaemia; ATG: Anti-thymocyte globulin; CB: Cord blood; $\mathrm{Cl}$ : Confidence interval; CK: Complex caryotype; CMV: Cytomegalovirus; CR1: First complete remission; CR2: Second complete remission; DLI: Donor leukocyte infusion; EBMT: European Society of Blood and Marrow Transplantation; EFS: Event-free survival; GRFS: GvHD-free/relapse-free survival; GvHD: Graft-versus-host disease; HLA: Human leukocyte antigen; HR: Hazard ratio; Inv(3): Inversion of chromosome 3; LFS: Leukaemia-free survival; MAC: Myeloablative conditioning; MK: Monosomal karyotype; NRM: Non-relapse mortality; OS: Overall survival; Rl: Relapse incidence; RIC: Reduced-intensity conditioning; TBI: Total body irradiation
\end{abstract}

\section{Acknowledgements}

Not applicable.

\section{Funding}

This retrospective study is supported by the EBMT.

\section{Availability of data and materials}

The dataset analysed in the present study is avalaible in the EBMT registry.

\section{Authors' contributions}

XP designed the study. XP and LM reviewed the cytogenetic data. XP, ML, JE and $A N$ analysed the data. XP wrote the paper. JE, MM and $A N$ reviewed the paper. JM, IYA, DB, NI, GS, TGD, NS, JC, SV and JS provide the data. JM, IYA, $\mathrm{DB}, \mathrm{NI}, \mathrm{GS}, \mathrm{TGD}, \mathrm{NS}, \mathrm{JC}, \mathrm{SV}$ and JS approved the manuscript. All authors read and approved the final manuscript.

\section{Competing interests}

The authors declare that they have no competing interests. 


\section{Consent for publication}

All patients included in the EBMT registry database have previously consented to be part anonymously to the registry.

\section{Ethics approval and consent to participate}

All retrospective studies performed by the EBMT are centrally approved by the EBMT board.

\section{Author details}

'Section of Hematology, Department of Medicine, Cliniques Universitaires Saint-Luc, 10, avenue Hippocrate, 1200 Brussels, Belgium. ${ }^{2}$ Acute Leukemia Working Party of the EBMT office, Paris, France. ${ }^{3}$ Department of Hematology, University Hospital Gasthuisberg, Leuven, Belgium. ${ }^{4}$ UAM allo-CSH, Hôpital HURIEZ, Lille, France. ${ }^{5}$ Programme de Transplantation et Thérapie Cellulaire, Centre de Recherche en Cancérologie de Marseille, Institut Paoli Calmettes, Marseille, France. ${ }^{6}$ Service des Maladies du Sang, CHRU, Angers, France. ${ }^{7}$ Department of Hematology, Hôpital Saint-Louis, Paris, France. ${ }^{8}$ Department of Medicine, Rikshospitalet, Oslo, Norway. ${ }^{9}$ Department of Hematology, Radboud University Medical Center, Nijmegen, The Netherlands. ${ }^{10}$ Daniel den Hoed Cancer Centre, Erasmus Medical Center, Rotterdam, The Netherlands. ${ }^{11}$ Service d'Hématologie, CHU Bordeaux, Pessac, France. ${ }^{12}$ Servicio de Hematologia, Hospital Universitario La Fe, Valencia, Spain. ${ }^{13} \mathrm{Center}$ for Human Genetics, KU Leuven and University Hospitals, Leuven, Belgium. ${ }^{14}$ Hematology department, IDIBAPS, Hospital Clinic, Barcelona, Spain. ${ }^{15}$ Service d'Hématologie clinique, Hôpital Saint-Antoine, AP-HP, Université Pierre et Marie Curie, INSERM UMRs U938, Paris, France. ${ }^{16}$ Chaim Sheba Medical Center, Tel-Hashomer, Israel.

Received: 8 October 2016 Accepted: 3 January 2017

Published online: 18 January 2017

\section{References}

1. Koreth J, Schlenk R, Kopecky KJ, et al. Allogeneic stem cell transplantation for acute myeloid leukemia in first complete remission: systematic review and meta-analysis of prospective clinical trials. JAMA. 2009;301:2349-61.

2. Cornelissen JJ, van Putten WL, Verdonck LF, et al. Results of a HOVON/SAKK donor versus no-donor analysis of myeloablative HLA-identical sibling stem cell transplantation in first remission acute myeloid leukemia in young and middle-aged adults: benefits for whom? Blood. 2007;109:3658-66.

3. Dohner H, Estey EH, Amadori S, et al. Diagnosis and management of acute myeloid leukemia in adults: recommendations from an international expert panel, on behalf of the European LeukemiaNet. Blood. 2010;115:453-74.

4. Grimwade D, Hills RK, Moorman AV, et al. Refinement of cytogenetic classification in acute myeloid leukemia: determination of prognostic significance of rare recurring chromosomal abnormalities among 5876 younger adult patients treated in the United Kingdom Medical Research Council trials. Blood. 2010;116:354-65.

5. Dohner H, Weisdorf DJ, Bloomfield CD. Acute myeloid leukemia. N Engl J Med. 2015;373:1136-52.

6. Grimwade D, Ivey A, Huntly BJ. Molecular landscape of acute myeloid leukemia in younger adults and its clinical relevance. Blood. 2016;127:29-41.

7. Schoch C, Kern W, Kohlmann A, et al. Acute myeloid leukemia with a complex aberrant karyotype is a distinct biological entity characterized by genomic imbalances and a specific gene expression profile. Genes Chromosomes Cancer. 2005:43:227-38.

8. Baron F, Labopin M, Niederwieser D, et al. Impact of graft-versus-host disease after reduced-intensity conditioning allogeneic stem cell transplantation for acute myeloid leukemia: a report from the Acute Leukemia Working Party of the European group for blood and marrow transplantation. Leukemia. 2012;26:2462-8.

9. Schlenk RF, Dohner K, Mack S, et al. Prospective evaluation of allogeneic hematopoietic stem-cell transplantation from matched related and matched unrelated donors in younger adults with high-risk acute myeloid leukemia: German-Austrian trial AMLHD98A. J Clin Oncol. 2010;28:4642-8.

10. Stelljes M, Beelen DW, Braess J, et al. Allogeneic transplantation as post-remission therapy for cytogenetically high-risk acute myeloid leukemia: landmark analysis from a single prospective multicenter trial. Haematologica. 2011;96:972-9.

11. Versluis J, Hazenberg CL, Passweg JR, et al. Post-remission treatment with allogeneic stem cell transplantation in patients aged 60 years and older with acute myeloid leukaemia: a time-dependent analysis. Lancet Haematol. 2015;2:e427-36
12. Ferrant A, Labopin M, Frassoni F, et al. Karyotype in acute myeloblastic leukemia: prognostic significance for bone marrow transplantation in first remission: a European Group for Blood and Marrow Transplantation study. Acute Leukemia Working Party of the European Group for Blood and Marrow Transplantation (EBMT). Blood. 1997;90:2931-8.

13. Cornelissen JJ, Breems D, van Putten WL, et al. Comparative analysis of the value of allogeneic hematopoietic stem-cell transplantation in acute myeloid leukemia with monosomal karyotype versus other cytogenetic risk categories. J Clin Oncol. 2012;30:2140-6.

14. Fang M, Storer B, Estey E, et al. Outcome of patients with acute myeloid leukemia with monosomal karyotype who undergo hematopoietic cell transplantation. Blood. 2011;118:1490-4.

15. Kayser S, Zucknick M, Dohner K, et al. Monosomal karyotype in adult acute myeloid leukemia: prognostic impact and outcome after different treatment strategies. Blood. 2012;119:551-8.

16. Brands-Nijenhuis AV, Labopin M, Schouten HC, et al. Monosomal karyotype as an adverse prognostic factor in patients with acute myeloid leukemia treated with allogeneic hematopoietic stem-cell transplantation in first complete remission: a retrospective survey on behalf of the ALWP of the EBMT. Haematologica. 2016;101:248-55.

17. Middeke JM, Beelen D, Stadler M, et al. Outcome of high-risk acute myeloid leukemia after allogeneic hematopoietic cell transplantation: negative impact of abnl(17p) and -5/5q. Blood. 2012;120:2521-8.

18. Harris CC, Hollstein M. Clinical implications of the p53 tumor-suppressor gene. N Engl J Med. 1993;329:1318-27.

19. Fenaux P, Preudhomme C, Quiquandon I, et al. Mutations of the P53 gene in acute myeloid leukaemia. Br J Haematol. 1992;80:178-83.

20. Wattel E, Preudhomme C, Hecquet B, et al. p53 mutations are associated with resistance to chemotherapy and short survival in hematologic malignancies. Blood. 1994;84:3148-57.

21. Fenaux P, Preudhomme C, Lai UL, et al. Cytogenetics and their prognostic value in de novo acute myeloid leukaemia: a report on 283 cases. Br J Haematol. 1989; 73:61-7.

22. Haferlach C, Dicker F, Herholz H, et al. Mutations of the TP53 gene in acute myeloid leukemia are strongly associated with a complex aberrant karyotype. Leukemia. 2008;22:1539-41.

23. Schiffer CA, Lee EJ, Tomiyasu T, et al. Prognostic impact of cytogenetic abnormalities in patients with de novo acute nonlymphocytic leukemia. Blood. 1989;73:263-70

24. Bowen D, Groves MJ, Burnett AK, et al. TP53 gene mutation is frequent in patients with acute myeloid leukemia and complex karyotype, and is associated with very poor prognosis. Leukemia. 2009;23:203-6.

25. Seifert H, Mohr B, Thiede $C$, et al. The prognostic impact of 17p (p53) deletion in 2272 adults with acute myeloid leukemia. Leukemia. 2009;23:656-63.

26. Grossmann V, Schnittger S, Kohlmann A, et al. A novel hierarchical prognostic model of AML solely based on molecular mutations. Blood. 2012:120:2963-72.

27. Middeke JM, Herold S, Rucker-Braun E, et al. TP53 mutation in patients with high-risk acute myeloid leukaemia treated with allogeneic haematopoietic stem cell transplantation. Br J Haematol. 2016;172:914-22.

28. Mohr B, Schetelig J, Schafer-Eckart K, et al. Impact of allogeneic haematopoietic stem cell transplantation in patients with abnl(17p) acute myeloid leukaemia. Br J Haematol. 2013;161:237-44.

29. Middeke JM, Fang M, Cornelissen JJ, et al. Outcome of patients with abnl(17p) acute myeloid leukemia after allogeneic hematopoietic stem cell transplantation. Blood. 2014;123:2960-7.

30. Rubio MT, Savani BN, Labopin M, et al. The impact of HLA-matching on reduced intensity conditioning regimen unrelated donor allogeneic stem cell transplantation for acute myeloid leukemia in patients above 50 years-a report from the EBMT acute leukemia working party. J Hematol Oncol. 2016;9:65.

31. Ruggeri A, Battipaglia G, Labopin M, et al. Unrelated donor versus matched sibling donor in adults with acute myeloid leukemia in first relapse: an ALWP-EBMT study. J Hematol Oncol. 2016;9:89.

32. Saraceni $F$, Labopin M, Gorin NC, et al. Matched and mismatched unrelated donor compared to autologous stem cell transplantation for acute myeloid leukemia in first complete remission: a retrospective, propensity scoreweighted analysis from the ALWP of the EBMT. J Hematol Oncol. 2016;9:79.

33. Schmid C, Schleuning $M$, Hentrich $M$, et al. High antileukemic efficacy of an intermediate intensity conditioning regimen for allogeneic stem cell transplantation in patients with high-risk acute myeloid leukemia in first complete remission. Bone Marrow Transplant. 2008;41:721-7. 
34. Rucker FG, Bullinger L, Schwaenen C, et al. Disclosure of candidate genes in acute myeloid leukemia with complex karyotypes using microarray-based molecular characterization. J Clin Oncol. 2006;24:3887-94.

35. Schmid C, Schleuning M, Ledderose G, et al. Sequential regimen of chemotherapy, reduced-intensity conditioning for allogeneic stem-cell transplantation, and prophylactic donor lymphocyte transfusion in high-risk acute myeloid leukemia and myelodysplastic syndrome. J Clin Oncol. 2005;23:5675-87.

36. Yan CH, Liu DH, Liu KY, et al. Risk stratification-directed donor lymphocyte infusion could reduce relapse of standard-risk acute leukemia patients after allogeneic hematopoietic stem cell transplantation. Blood. 2012;119:3256-62.

37. Bally C, Ades $L$, Renneville $A$, et al. Prognostic value of TP53 gene mutations in myelodysplastic syndromes and acute myeloid leukemia treated with azacitidine. Leuk Res. 2014;38:751-5.

38. Goodyear OC, Dennis M, Jilani NY, et al. Azacitidine augments expansion of regulatory $T$ cells after allogeneic stem cell transplantation in patients with acute myeloid leukemia (AML). Blood. 2012;119:3361-9.

39. Platzbecker U, Wermke M, Radke J, et al. Azacitidine for treatment of imminent relapse in MDS or AML patients after allogeneic HSCT: results of the RELAZA trial. Leukemia. 2012;26:381-9.

40. Craddock C, Labopin M, Robin M, et al. Clinical activity of azacitidine in patients who relapse after allogeneic stem cell transplantation for acute myeloid leukemia. Haematologica. 2016;101 (7):879-83.

41. Lim O, Jung MY, Hwang YK, et al. Present and future of allogeneic natural killer cell therapy. Front Immunol. 2015;6:286.

42. Pittari G, Filippini $P$, Gentilcore $G$, et al. Revving up natural killer cells and cytokine-induced killer cells against hematological malignancies. Front Immunol. 2015;6:230.

43. Stern M, Passweg JR, Meyer-Monard S, et al. Pre-emptive immunotherapy with purified natural killer cells after haploidentical SCT: a prospective phase II study in two centers. Bone Marrow Transplant. 2013;48:433-8.

44. Hotinski AK, Lewis ID, Ross DM. Vosaroxin is a novel topoisomerase-ll inhibitor with efficacy in relapsed and refractory acute myeloid leukaemia. Expert Opin Pharmacother. 2015:16:1395-402.

45. Walsby EJ, Coles SJ, Knapper S, et al. The topoisomerase II inhibitor voreloxin causes cell cycle arrest and apoptosis in myeloid leukemia cells and acts in synergy with cytarabine. Haematologica. 2011;96:393-9.

46. Castro PD, Liang JC, Nagarajan L. Deletions of chromosome $5 q 13.3$ and 17p loci cooperate in myeloid neoplasms. Blood. 2000;95:2138-43.

47. Kulasekararaj AG, Smith AE, Mian SA, et al. TP53 mutations in myelodysplastic syndrome are strongly correlated with aberrations of chromosome 5, and correlate with adverse prognosis. Br J Haematol. 2013;160:660-72.

48. Stoddart A, Fernald AA, Wang J, et al. Haploinsufficiency of del(5q) genes, Egr1 and Apc, cooperate with Tp53 loss to induce acute myeloid leukemia in mice. Blood. 2014:123:1069-78.

49. Bejar R, Stevenson K, Abdel-Wahab O, et al. Clinical effect of point mutations in myelodysplastic syndromes. N Engl J Med. 2011:364:2496-506.

50. Shih AH, Chung SS, Dolezal EK, et al. Mutational analysis of therapy-related myelodysplastic syndromes and acute myelogenous leukemia. Haematologica. 2013;98:908-12.

51. Walter MJ, Shen D, Shao J, et al. Clonal diversity of recurrently mutated genes in myelodysplastic syndromes. Leukemia. 2013;27:1275-82.

\section{Submit your next manuscript to BioMed Central and we will help you at every step:}

- We accept pre-submission inquiries

- Our selector tool helps you to find the most relevant journal

- We provide round the clock customer support

- Convenient online submission

- Thorough peer review

- Inclusion in PubMed and all major indexing services

- Maximum visibility for your research

Submit your manuscript at www.biomedcentral.com/submit

) Biomed Central 